DYMAT 2009 (2009) 1109-1115

(C) EDP Sciences, 2009

DOI: $10.1051 /$ dymat/2009155

\title{
The effect of impact compaction on surface quality of powder discs
}

P. Jonsén ${ }^{1}$, H. Häggblad ${ }^{1}$ and M. Nishida ${ }^{2}$

1 Luleå University of Technology, 97187 Luleå, Sweden

2 Nagoya Institute of Technology, Gokiso-cho, Showa-ku, Nagoya, Japan

\begin{abstract}
Powder compaction by impact where high strain-rates occurs in the powder is attending an increasing interest. Impact compaction or high velocity compaction (HVC) is a production technique with capacity to significantly improve the mechanical properties of powder metallurgy (PM) parts. The HVC experiments were performed using a laboratory machine with a hydraulically driven hammer. This machine has maximum impact energy of $4 \mathrm{~kJ}$. The powder materials used for the investigation were pre-alloyed water atomized iron based powders. Profile measurements of HVC and conventionally compacted (CC) $25 \mathrm{~mm}$ circular discs have been performed with a Wyko NT1100 using vertical scanning interferometry (VSI). For comparable densities the HVC discs have a significantly flatter and smoother upper surface (impact face) compared to $\mathrm{CC}$ discs.
\end{abstract}

\section{INTRODUCTION}

Powder metallurgy (PM) is a rapidly developing technology. Economic advantages such as high material utilization ( $95 \%$ of the raw material is used in the final product, in comparison with as little as $50 \%$ in other forming processes) and low energy consumption make it a particularly attractive forming process. The PM process can also produce the unique microstructures and compositions required for cemented carbides and other hard materials, refractory metals, magnets, and composite materials.

High velocity compaction (HVC) of particulate materials or powder systems is a relatively new mass production technique with the capacity significantly to improve the mechanical properties, and hence expand the applications of powder materials parts. An overview of the process and its capabilities, and potential applications related to PM are discussed in [1]. A punch impacting into a powder compact in a die will generate elastic and plastic waves of deformation propagating through the compact, causing impacts between particles and consolidation of the compact. A number of investigations of the HVC process have been published recently. All publications indicate that high-density components can be obtained using HVC [2-7]. Other characteristics are low spring back, low ejection forces and uniform densities. This paper describes an experimental investigation with the goal to compare the two processes HVC and Conventional Compaction (CC) regarding shape effects. Dimension control and surface quality are important aspects in many forming applications, this is also important for the HVC process. Great care has been taken to compare these two processes under the same conditions, i.e. tooling set up, powder material, lubricant, etc.

The global objective of our research is to improve the fundamental understanding of the mechanics of compaction. For all materials, densification occurs as a result of contact interactions between the particles. The details of these interactions are, however, different for each individual material. 


\section{EXPERIMENTAL PROCEDURE}

In order to compare HVC with conventional quasi static compaction, the experiments have been performed under the same conditions. During the different experiments the tool set, powder material, force sensor, data acquisition equipment and sample geometry were identical. The tool set used during the experiments consists of a top punch, bottom punch and a die. The upper and bottom punch are manufactured of high speed sinter steel. The die contains a shrink fit pretensioned hard metal insert. The diameter of both punches was $25 \mathrm{~mm}$ and the radial clearance between punches and die was $0.010-0.015 \mathrm{~mm}$. The height of the top and bottom punch was $80 \mathrm{~mm}$ and $30 \mathrm{~mm}$, respectively, the height of the die $90 \mathrm{~mm}$.

\subsection{Metal powder material}

The powder material used for the investigation was a press-ready mix containing Astaloy Mo $+0.3 \%$ graphite (uf 4 ) $+0.6 \%$ Kenolube. Astaloy Mo is a pre-alloyed water atomized iron powder with 1.5\% Molybdenum, from Höganas AB Sweden. Kenolube is a lubricant from the same supplier. The theoretical pore free density of this mix is $7.45 \mathrm{~g} / \mathrm{cm}^{3}$. Measurements were also performed for a press-ready mix containing Distaloy AE $+0.5 \%$ graphite (uf- 4$)+0.6 \%$ Kenolube with the theoretical pore free density of $7.48 \mathrm{~g} / \mathrm{cm}^{3}$.

\subsection{High Velocity Compaction}

The High Velocity Compaction experiments were performed using a laboratory HVC-machine with a hydraulically driven hammer. This machine has a maximum capacity of $4 \mathrm{~kJ}$. The hydraulic hammer consists of two parts: the hydraulic piston and a weight, which is connected to the piston rod. The hydraulic oil pressure applied on the hydraulic piston is constant and thereby also the force accelerating the piston and the weight. The total mass accelerated was $31 \mathrm{~kg}$. See Figure 1 for a schematic view.

Due to the constant acting force, the energy level is easily adjustable by varying the acceleration distance of the hammer, i.e. the distance between the start position and the impact position. The level of energy can be calculated by multiplying the accelerating force and the distance. Further, the machine was equipped with a load-cell, located under the bottom punch. Load cell data were acquired by a high speed measuring system, capable to measure very sharp and short peak loads due to the impact, see e.g. [3]. The sampling rate in this case was $200 \mathrm{kHz}$. The HVC process starts typically with a pre-compaction, during which the powder is compacted from the apparent density to a density of approximate $5 \mathrm{~g} / \mathrm{cm}^{3}$ and thereby the main amount of air is evacuated from the cavities. During the HVC experiments the die was mechanically fixed, i.e. the die was not floating.

The impact compaction force curve was measured during each HVC experiment. A single HVC compaction results in several force peaks, see Figure 2. This is because the hydraulic hammer bounces on the top punch until the impact energy dissipates. The hammer impacts the upper punch with its mass and the residual oil pressure in the accumulator until stress is more or less stabilized. The maximum compaction pressure occurs in the first and highest force peak. The experiment shown in Figure 2 has an impact velocity of $13.2 \mathrm{~m} / \mathrm{s}$ corresponding to an impact energy of $2.7 \mathrm{~kJ}$ and a peak force of $776 \mathrm{kN}$ with a maximum compaction pressure of $1581 \mathrm{MPa}$. The resultant relative density for this test is 0.993 . The maximum overall strain-rate is of the order $10^{2} \mathrm{~s}^{-1}$ for the experiments done. 


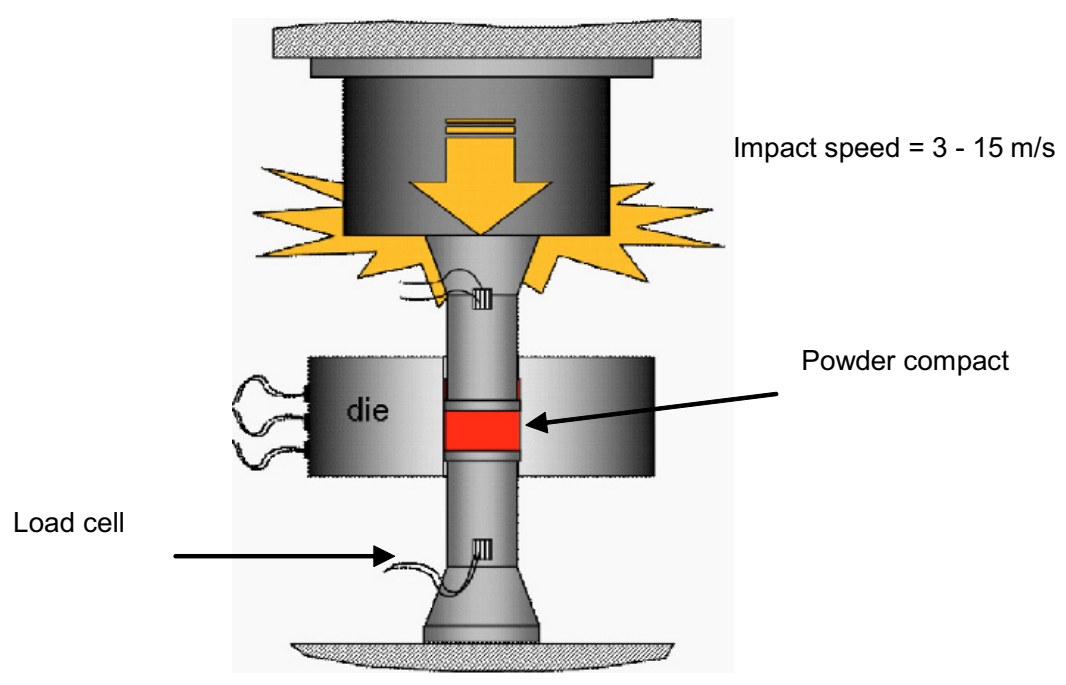

Figure 1. Schematic view of High Velocity Compaction (HVC) [7].
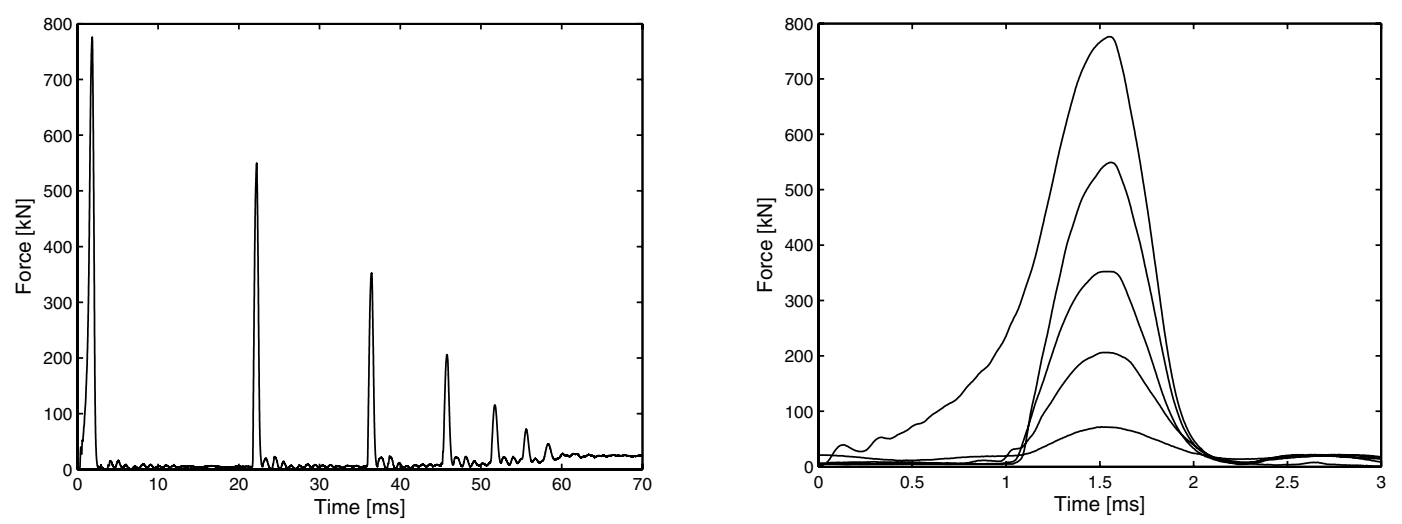

Figure 2. HVC force curve measured during a high velocity compaction experiment with impact energy of $2.7 \mathrm{~kJ}$, (a) complete curve, (b) peaks only.

\subsection{Conventional Compaction}

The Conventional Compaction experiments were performed using a manual 40 ton workshop press. During CC the compaction force was measured with the load cell also used for the HVC experiments. The load-cell was placed under the bottom punch. The compaction time for a CC test was approximately 5 seconds.

\subsection{Vertical Scanning Interferometry and topographical measurement}

Profile measurements of HVC and CC pressed circular discs have been performed with a Wyko NT1100 using vertical scanning interferometry (VSI). The technique uses the bright and dark pattern which is a result of the splitting of a beam where one part is reflected against an internal reference surface and the other of the sample. After reflection the beams recombine in the interferometer and the patterns of constructive and destructive interference occur. Each scanning covers an area of $3.8 \times 5.0 \mathrm{~mm}^{2}$. To measure a disc multiple scans are performed and the results 
are stitched together. An overlap of $20 \%$ is used in each scan and the pixel size is $13.14 \mu \mathrm{m}$ in the horizontal-plane. The vertical resolution is $3 \mathrm{~nm}$ with maximum error less than $0.5 \%$.

The powder is compacted into $25 \mathrm{~mm}$ discs with a thickness of $5 \mathrm{~mm}$ and all surfaces of the specimen are scanned, see Figure 3. To estimate the profile shape and the surface roughness of the upper and lower surfaces from the scanned data a cross hair cursor with a width of $10 \%$ of the diameter is used, see Figure 3. From the two paths the profile shape and the roughness are found.
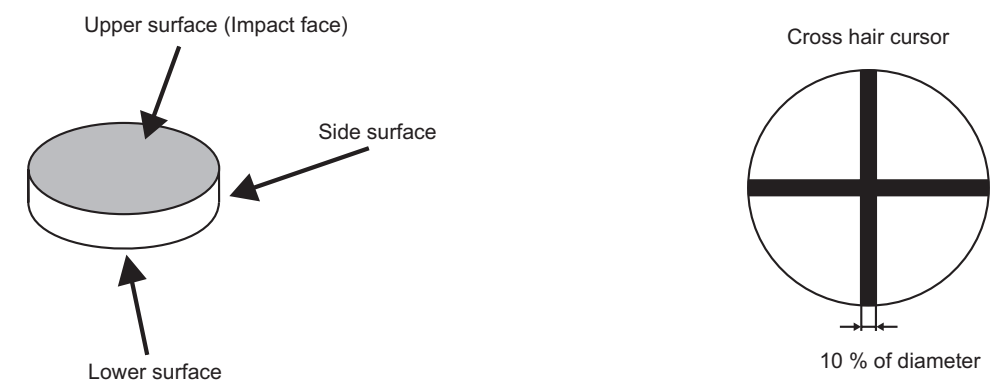

Figure 3. Surfaces scanned with VSI. The scanned data is evaluated with a cross hair cursor.

\section{RESULTS}

\subsection{Analysis of the HVC force curve}

The increase of compacting force and the duration will lead to higher densification of the powder body. The densification is achieved by a force created by a hydraulically driven hammer. This transfers the kinetic energy through the upper punch to the powder compact. As stress waves propagate within the powder body, interactions cause transmission and reflection, which lead to further densification of the powder. The HVC process in this study resulted in a higher compressibility compared to $\mathrm{CC}$, that is, for the same maximum pressure a higher density is reached for HVC than CC. See [6] where this is described in more detail.

In Figure 2 (b) the force versus time is shown for the first impact and the following four bouncing impacts. Each impact consists of a loading and an unloading phase. The loading phase may be a combination of elastic and plastic waves, while the unloading is purely elastic. The first impact shows a slowly increasing and lower maximum force rate during loading than during unloading, while the following impacts are fairly symmetric, that is, approximately the same maximum force rate during the loading as during unloading phase. This suggests that most of the rearrangement and plastic deformation of the powder particles occurs during the loading phase of the first impact. The shape of the force curve shows that further densification occurs at the end of the loading face during the second and third impact; see Figure 2 (b). The characteristic time of impacts two to five is approximately $1 \mathrm{~ms}$ which corresponds well with an eigenfrequency analysis.

\subsection{Surface profile}

The surface profiles of $25 \mathrm{~mm}$ diameter and $5 \mathrm{~mm}$ high $\mathrm{HVC}$ and CC discs are compared. The pressing has applied a single sided action with the top punch during compaction followed by a downward ejection. Results from the profile measurements for CC and HVC discs compacted to the same density and a HVC disc with maximum density are shown in Figure 4. Both upper- and lower surfaces of HVC- and CC discs have a dished profile. For comparable densities the HVC discs have a flatter upper surface, about $6 \mu \mathrm{m}$ difference in height from the centre to the edge of the 
disc compared to $18 \mu \mathrm{m}$ for CC discs, see Figure 4 (a) and Figure 4 (b). On the lower surface, CC discs seems to have a deeper dish, $35 \mu \mathrm{m}$ compared to $25 \mu \mathrm{m}$ for HVC, but no large differences between HVC and CC pressed discs are found regarding the lower surface. For HVC discs manufactured with high-energy impact and densities close to the theoretical pore free density the upper- and lower surfaces of HVC discs appear to have a dome, see Figure 4 (c). The force curve resulting in the shape of Figure 4 (c) is shown in Figure 2.

For CC with its slow compaction speed the powder grains rearrange as the compaction pressure is increasing. Powder grains move in the radial direction and the radial pressure rises. For HVC the impacted face moves at high speed. The time for rearrangement in the powder grain is short and inertia of the powder mix will limit the movement and thereby the magnitude of the radial pressure. This can explain the difference of the impact face between HVC and CC.

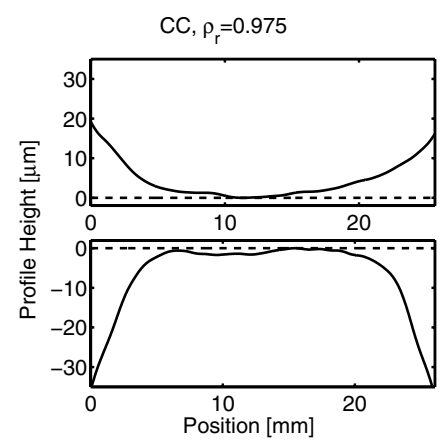

(a)

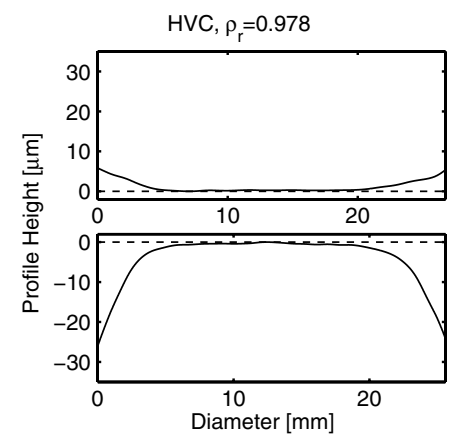

(b)

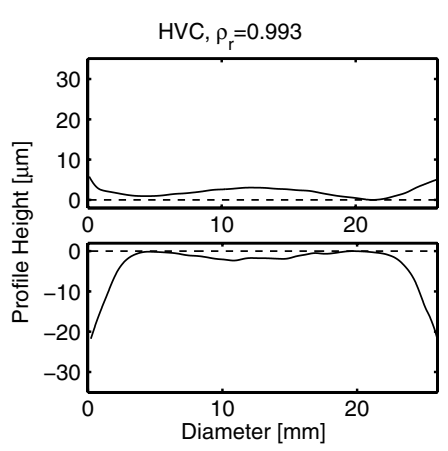

(c)

Figure 4. Profile across the diameter of the upper surface and the lower surface. (a) Conventionally pressed. (b) HVC pressed. (c) HVC compact with maximum density.

\subsection{Surface texture}

The arithmetic average of the roughness profile $\left(R_{a}\right)$ and the maximum height of the profile $\left(R_{t}\right)$ are measured with the results shown in Figure 5. No differences between the two powder mixes could be found, Figure 5 contains results from both mixes. Even though the results are for a limited number of compacts, conclusions could be drawn. The HVC compacts have a smaller $\mathrm{R}_{\mathrm{a}}$ and $R_{t}$ on both sides especially for the upper surface (impact face) where $R_{a}$ is three times better for HVC samples compared to CC samples.

The side surface of the compact is also measured. The results show no significant difference between HVC and CC compacts. The $\mathrm{R}_{\mathrm{a}}$-value is below $1 \mu \mathrm{m}$ and the side surface has an hourglass shape with a depth of 2-4 $\mu \mathrm{m}$.

The data of a scanned surface assembles a three dimensional landscape which can be used to investigate the topography of the impacted surface. Figure 6 shows the surface of a high-energy impacted HVC manufactured disc corresponding to Figure 4 (c). The figure reveals a flat surface with a dome in the centre.

\section{CONCLUSIONS}

In order to compare the HVC and CC processes as identical conditions as possible were applied. The main difference between the processes in this study is the velocity of the top punch and the several very sharp and short peak loads during a single HVC impact. 


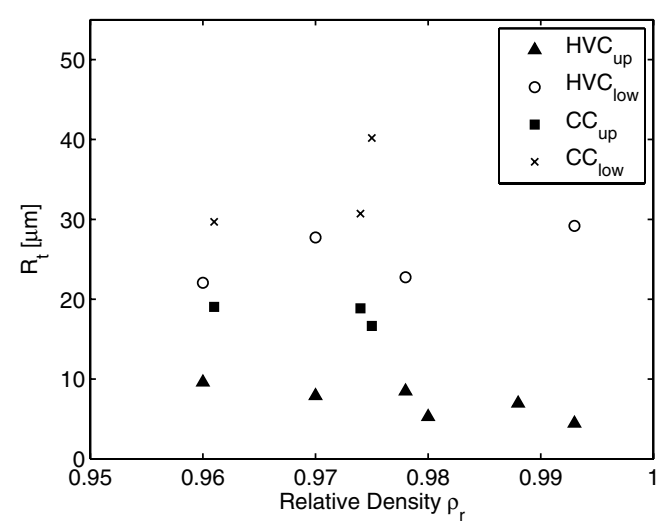

(a)

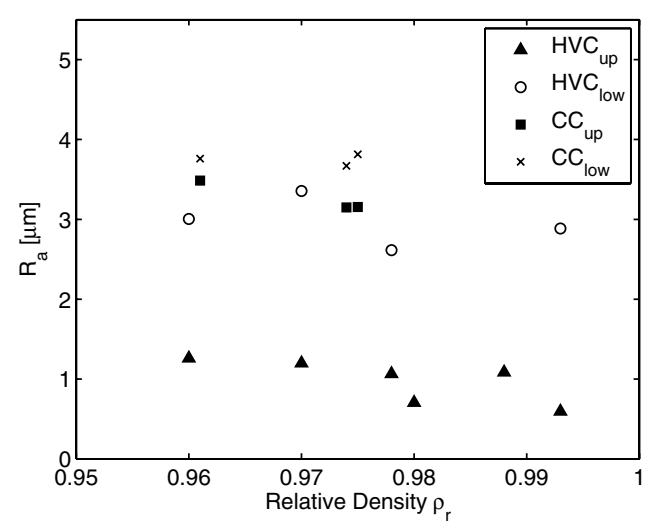

(b)

Figure 5. The arithmetic average of the roughness profile $\left(R_{a}\right)$ and the maximum height of the profile $\left(R_{t}\right)$ for different relative densities. The upper surface (up) and the lower surface (low) for both HVC and conventionally pressed compacts.

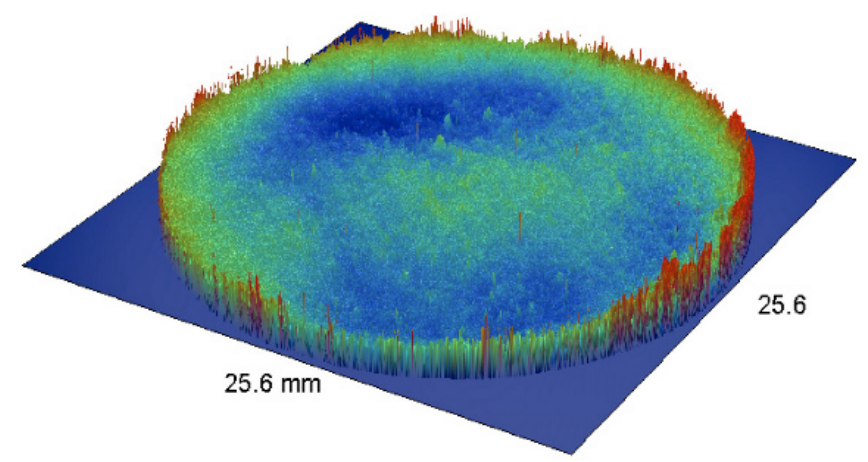

Figure 6. The three dimensional data of the scanned upper surface of a high energy impacted HVC disc. The differences between the highest and lowest point in the impact surface is $10 \mu \mathrm{m}$. The corresponding twodimensional profile is shown in Figure 4 (c).

Both HVC and CC samples have dish shaped end surfaces. The measurement of the texture of the surfaces shows that the surface profile of a HVC sample is flatter and smoother than for a CC sample. The arithmetic average of the roughness profile $\left(R_{a}\right)$ is approximately one third for the impact face of HVC green compacts than those produced by conventional compaction with the same density.

\section{Acknowledgments}

Tomas Dahllöf at MeTec Powder Metal AB is acknowledged for his support of manufacturing the test samples.

\section{References}

[1] Skoglund P., Powder Metallurgy, Vol. 44, No 3, (2001), 199.

[2] Doremus P., Duwa F., Francois P., Puente G. and Allibert C., High Velocity Compaction, Proceedings PM2TECH 2002, Vol. 4, MPIF, Orlando, USA, (2002), 96. 
[3] Skagerstrand A., High Velocity Compaction of Metal Powders, a Study on Density and Properties, Proceedings PM2TECH 2002, Vol. 4, MPIF, Orlando, USA, (2002), 111.

[4] Ericsson T. and Luukkonen P., Materials Science Forum, 407 (2002) 77-82.

[5] Sethi G., Hauck E. and German R., Materials Science and Technology. 22 (2006) 955-959.

[6] Jonsén P., Häggblad H., Troive L., Furuberg J., Allroth S. and Skoglund P., Materials Science Forum, Trans Tech publications (2007), 289-292.

[7] Dore F., Lazzarotto L. and Bourdin S., Materials Science Forum, Trans Tech publications (2007), 293-296. 